\title{
DESEMPENHO DE INDIVÍDUOS COM DERMOPIGMENTAÇÃO SUBMETIDOS À PRÁTICA DE EXERCÍCIO FÍSICO
}

\author{
PERFORMANCE OF DERMAL PIGMENTED INDIVIDUALS SUBMITTED TO PHYSICAL EXERCISE \\ Artigo Original \\ Original ARTICLE \\ DESEMPEÑO DE INDIVIDUOS CON DERMOPIGMENTACIÓN SOMETIDOS A PRÁCTICA DE EJERCICIOS FÍSICOS \\ Artículo Original
}

Marco Antonio Uzunian

(Educador Físico)

Marcelo Luis Marquezi

(Educador Físico)

Bruno Leonardo Ugolini

(Educador Físico)

Adriana Tedim Bimbato ${ }^{1}$

(Educadora Física)

Felipe Borges Trama

(Educador Físico)

Marcel Eduardo Vicaria Pinotti

(Educador Físico)

Thiago Soares Camargo Póca'

(Educador Físico)

Lucas Duarte Tavares'

(Educador Físico)

1. Universidade Cidade de São Paulo (UNICID), Laboratório de Pesquisa em Educação Física e Fisioterapia), São Paulo, SP, Brasil.

\section{Correspondência:}

Rua Cel. Irineu de Castro, 131, Jardim Anália Franco, São Paulo, SP, Brasil. 03333-050.uzunian@ig.com.br

\section{RESUMO}

Introdução: A duração e a intensidade dos exercícios provocam diferentes alterações no sistema imunológico, dentre elas a diminuição das respostas imunes, acarretando queda no desempenho físico. Sabe-se também que a dermopigmentação pode colaborar com tais alteraç̃̃es no comportamento deste sistema. Objetivo: Avaliar os efeitos da dermopigmentação sobre o desempenho no exercício físico. Método: Neste ensaio clínico participaram 12 sujeitos, dentre eles seis com dermopigmentação, idade (anos), peso (Kg), estatura (cm) e gordura corporal (\%) de 16,5 $\pm 1,16$; $68,10 \pm 6,83 ; 176,29 \pm 7,71 ; 11,49 \pm 0,58$ respectivamente, divididos em dois grupos, sendo submetidos a teste de esforço máximo com intensidade progressiva em esteira ergométrica para determinação do consumo máximo de oxigênio (VO2 máx), limiares anaeróbios (LAns) e economia de corrida (EC 14km/h\%), sendo realizadas coletas de lactato pré e 5 minutos pós-teste. Os dados foram tratados por "teste $t$ " para variáveis independentes, tendo como nível de significância adotado $a<0,05$. Resultados: Foram observadas diferenças significativas entre os grupos em relação à concentração sanguínea de lactato (CSL) pré-teste $(4,6 \pm 0,11$ e 3,23 \pm 0,88 para dermopigmentados e não dermopigmentados, respectivamente; $p<0,05)$ e 5 minutos pós-teste $(5,65 \pm 0,49$ e 13,13 $\pm 2,20$ para dermopigmentados e não dermopigmentados, respectivamente; $p<0,05)$. O tempo de teste também foi significativamente superior para o grupo não dermopigmentado em comparação ao grupo com dermopigmentação (8 min 47 seg $\pm 0,08$ e 7 min 39 seg $\pm 0,03$ respectivamente; $p<0,05)$, bem como análise do WURSS-21 (3,33 $\pm 3,14$ e 2,00 $\pm 1,09$, respectivamente; $p$ $>0,05)$. Em relação às demais variáveis dependentes ( $\mathrm{VO}_{\text {máx }}$ segundo limiar anaeróbio e economia de corrida) não foram encontradas diferenças estatisticamente significativas. Conclusão: A dermopigmentação produziu rendimento inferior no teste de esforço máximo, influenciando o desempenho no exercício físico.

Descritores: esforço físico, tatuagem, imunidade.

\section{ABSTRACT}

Introduction: Duration and intensity of exercises cause different alterations in the immunity system, among them, reduction of immunity responses, causing decrease in physical performance. It is also known that dermal pigmentation can influence such alterations in the system's behavior. Objective: To evaluate the effects of dermal pigmentation on performance of physical exercise. Method: In this clinical essay, 12 subjects participated, among them, 6 with dermal pigmentation, being age (years), weight ( $\mathrm{kg})$, height (cm) and body fat (\%) measured as $16.5 \pm 1.16 ; 68.10 \pm$ $6.83 ; 176.29 \pm 7.71 ; 11.49 \pm 0.58$, respectively, divided in two groups. They were submitted to a maximum effort test with gradual intensity in treadmill to determine maximum oxygen consumption (VO2max), anaerobic thresholds (AnT) and running economy (RE $14 \mathrm{~km} / \mathrm{h} \%$ ), lactate being collected before and 5 minutes after test. Data has been analyzed by "t test" for independent variables, having a level of significance of $a<0.05$. Results: Significant differences were observed between groups in relation to pre-test blood lactate concentration (BLC) (4.6 \pm 0.11 and $3.23 \pm 0.88$ for the dermal pigmented and non-dermal pigmented groups, respectively; $p<0.05)$ and 5 minutes post-test $(5.65$ \pm 0.49 and $13.13 \pm 2.20$ for the dermal pigmented and non-dermal pigmented groups, respectively; $p<0.05)$. The testing time was also significantly higher for the non-dermal pigmented group, compared to the dermal pigmented group (8 $\mathrm{min} 47 \mathrm{sec} \pm 0.08$ and $7 \mathrm{~min} 39 \mathrm{sec} \pm 0.03$ respectively; $p<0.05$ ) and also analysis of WURSS-21 $3.33 \pm$ 3.14 and $2.00 \pm 1.09$, respectively; $p>0.05$ ). In relation to the other dependent variables (VO2max, second anaerobic threshold and running economy) no statistically significant differences were found. Conclusion: Dermal pigmentation produced lower performance in the maximum effort test, with an influence on performance of physical exercise.

Keywords: physical exertion, tattooing, immunity.

\section{RESUMEN}

Introducción: La duración e intensidad del ejercicio provocan diferentes cambios en el sistema inmunológico, entre ellos, la reducción de las respuestas inmunes, lo que resulta en disminución en el rendimiento físico. También se sabe que la dermopigmentación puede colaborar con tales cambios en el comportamiento de este sistema. Objetivo: Evaluar los efectos de dermopigmentación en el rendimiento del ejercicio físico. Método: En este ensayo clínico participaron 12 sujetos, entre ellos seis con dermopigmentación, con edad (años), peso (kg), altura (cm) y grasa corporal (\%) de $16,5 \pm 1,16 ; 68,10 \pm 6,83 ; 176,29 \pm 7,71 ; 11,49 \pm 0,58$, respectivamente, divididos en dos grupos. Estos se sometieron a la prueba de esfuerzo máximo con intensidad progresiva en una cinta ergométrica para determinar el consumo máximo

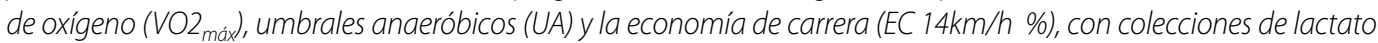
pre y 5 minutos después de la prueba. Los datos fueron analizados por "prueba t" para las variables independientes, teniendo un nivel de significación adoptado de $a<0,05$. Resultados: Se observaron diferencias significativas entre los 
grupos con respecto a la concentración de lactato en sangre (CLS) pre-test $(4,6 \pm 0,11$ y 3,23 $\pm 0,88$ para dermopigmentados y no dermopigmentados, respectivamente; $p<0,05)$ y 5 minutos después de la prueba $(5,65 \pm 0,49$ y 13,13 $\pm 2,20$ para dermopigmentados no dermopigmentados, respectivamente; $p<0,05)$. El tiempo de prueba fue también significativamente mayor para el grupo no dermopigmentado en comparación con el grupo con dermopigmentación (8 min $47 \mathrm{seg} \pm 0,08$ y 7 min $39 \mathrm{seg} \pm 0,03$, respectivamente; $p<0,05)$ y WURSS-21 análisis $(3,33 \pm 3,14$ y 2,00 \pm 1,09 respectivamente; $p>0,05)$. En cuanto las demás variables dependientes ( $\mathrm{VO} 2_{\text {máx }}$ segundo umbral anaeróbico y economía de carrera) no se encontraron diferencias estadísticamente significativas. Conclusión: La dermopigmentación produjo bajo rendimiento en la prueba de esfuerzo máximo, que influye en el rendimiento del ejercicio físico.

Descriptores: esfuerzo físico, tatuaje, inmunidad.

\section{INTRODUÇÃO}

O uso da dermopigmentação é tão antigo quanto à própria civilização. Acredita-se que ela pode ter surgido em várias partes do mundo de maneira independente e sido difundida através das navegações realizadas pelos países europeus. Atualmente, estima-se que nos Estados Unidos (EUA) e Europa existam mais de 100 milhões de indivíduos portadores de dermopigmentação'.

Em termos práticos, a dermopigmentação trata-se de uma aplicação subcutânea conseguida através da introdução de pigmentos por intermédio de agulhas, bem como máquinas de alta rotação ${ }^{1,2}$. Entre os pigmentos mais utilizados em sua realização figuram o carbono, sulfeto de mercúrio, tintas vegetais, cobalto, sulfeto de cádmio, óxido de cromo, ocre e óxido de ferro ${ }^{2}$. Durante sua confecção as agulhas penetram na pele cerca de 1,5 milímetros de profundidade com frequência de perfuração da pistola de $3.000 \mathrm{~Hz}$.

Tal procedimento é responsável por gerar micro lesões nos tecidos envolvidos, estimulando o organismo a liberar substâncias endógenas com a função de reparar estas estruturas ${ }^{1}$.

Diante disto, a pele e sua complexa estrutura tecidual, além de revestir o organismo, também isola componentes orgânicos do meio exterior, sendo responsável pela detecção de estímulos aferentes e que através do sistema imune proporciona uma significativa proteção aos tecidos impedindo a entrada de substâncias no organismo por intermédio de células imunologicamente ativas presentes na derme, onde intensamente atuam componentes da imunidade celular e humoral ${ }^{3}$. Logo, qualquer alteração da função ou do aspecto da pele pode gerar consequências nocivas à saúde física, como: infecções repetidas e reações anormais e alérgicas.

Durante o processo de confecção da dermopigmentação foram relatados alguns distúrbios orgânicos imediatos à mesma, sendo eles: fome, sonolência, relaxamento, calafrio, febre, tensão muscular, necessidade de defecar, irritabilidade e fadiga. Além destes, após o processo de confecção, foram relatados sintomas de fadiga, relaxamento, dispepsia e algias osteomioarticulares ${ }^{1}$.

Como visto anteriormente, o sistema imune inclui um grupamento altamente complexo e auto-regulador de células, hormônios e moduladores interativos que defendem o corpo contra a invasão de micróbios provenientes do meio externo além de possíveis distúrbios orgânicos que venham a ocorrer ${ }^{4-7}$. Estudos demonstraram que este sistema, quando relacionado ao exercício físico como modelo mensurável de indução ao estresse também sofre alterações funcionais consideráveis ${ }^{6,8-11}$.

A duração e a intensidade dos exercícios provocam diferentes alterações nos parâmetros deste sistema. A literatura tem apresentado evidências de que o exercício físico moderado $\left(<60 \%\right.$ do $\left.\mathrm{VO}_{2 \text { máx }}\right)$ pode estar relacionado com o aumento da resposta imune dos mecanismos de defesa do organismo por aumento nas concentrações de glutamina plasmática, sendo este aminoácido produzido pelo metabolismo oxidativo durante atividades com esta intensidade ${ }^{12-17}$.

Entretanto, exercícios de alta intensidade e longa duração (>65\% do $\mathrm{VO}_{2 \text { máx }}$ ) e treinamento excessivo parecem diminuir as respostas imunes pois a glutamina passa a ser convertida em glicose por intermédio do fígado através do processo de neoglicogênese, contribuindo com o metabolismo glicolítico para a manutenção destas atividades, deixando então de atuar junto ao sistema imunológico ${ }^{13,18-20}$.

Portanto, qualquer alteração da função ou do aspecto da pele pode gerar consequências nocivas à saúde física com consequente participação do sistema imune no combate a tais alterações ${ }^{3}$. Além disso, sabe-se que o exercício físico como um dos fatores de indução ao estresse também contribui para que ocorram alterações funcionais neste sistema ${ }^{6,9,10,11}$.

O objetivo do presente estudo foi avaliar os efeitos da dermopigmentação sobre o desempenho no exercício físico.

\section{MÉTODO}

Todos os procedimentos foram submetidos e aprovados pelo Comitê de Ética em Pesquisa Envolvendo Seres Humanos da Universidade Cidade de São Paulo, São Paulo, SP, Brasil (Protocolo no 13537946). Os sujeitos assinaram o Termo de Consentimento Livre e Esclarecido referente à participação após concordarem com os objetivos, riscos e benefícios relacionados ao estudo. As intervenções referentes ao estudo em questão foram realizadas nas dependências da Universidade Cidade de São Paulo, tendo seus experimentos metodológicos conduzidos no Laboratório de Pesquisa em Educação Física e Fisioterapia (LAPEFFI).

\section{Casuística}

Para a realização deste ensaio clínico foram recrutados 12 sujeitos (jogadores da categoria sub-17 de uma equipe profissional de futebol de campo). Dentre eles, seis dermopigmentados, com área dermopigmen-

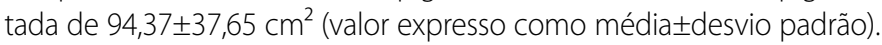

Os sujeitos foram divididos em dois grupos com seis indivíduos cada, de acordo com os seguintes critérios de inclusão: serem atletas, portadores de única tatuagem, dermopigmentados há mais de 1 ano, assintomáticos, não fumantes e que não utilizam qualquer medicamento. Como critérios de exclusão foram considerados: atletas com duas ou mais tatuagens, em recuperação de lesão, com histórico familiar de cardiopatia, diabetes e/ou portadores das mesmas.

Foram realizadas avaliações clínica (exame médico) e física (avaliação antropométrica e composição corporal). Para a caracterização do exercício físico em questão, foi utilizado o teste de esforço máximo em esteira ergométrica com o objetivo de coletar dados referentes ao consumo máximo de oxigênio $\left(\mathrm{VO}_{2 \text { máx }}\right)$, economia de corrida (EC14Km/h), limiares anaeróbios (LAn1 e LAn2) e coletas de lactato pré e pós-teste.

Ao final do teste, 10 minutos após a realização do mesmo, todos os indivíduos responderam ao questionário WURSS-21. Por intermédio deste, pretendia-se monitorar as alterações no esforço percebido em cada sessão, fadiga, percepção de sintomas de estresse e detecção de infecções do trato respiratório superior (ITRS). O fluxograma abaixo detalha o delineamento experimental do presente estudo (Figura 1).

Os procedimentos da coleta de dados foram realizados em uma única sessão experimental. Anteriormente à realização das avaliações/ 


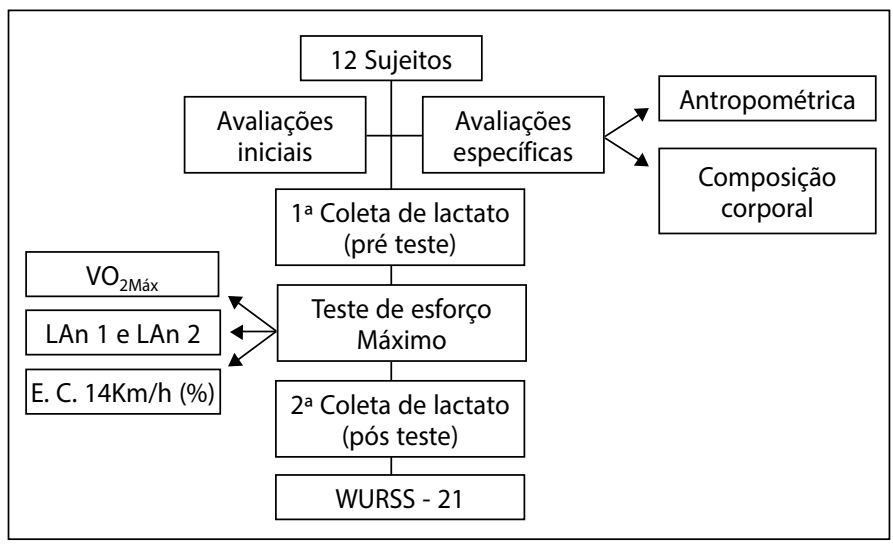

Figure 1. Delineamento experimental do estudo.

testes, todos os equipamentos utilizados na aquisição, armazenamento e processamento dos sinais biológicos foram calibrados. Em cada sessão experimental foram testados dois sujeitos.

Estes sujeitos receberam orientação prévia para não realizarem qualquer tipo de esforço físico extenuante, não ingerir bebidas alcoólicas e/ou estimulantes (chá, café) no dia anterior as sessões, tendo o recordatório alimentar do dia e também anterior aos testes, analisado (estes não interferiram no rendimento dos grupos).

Após avaliação clínica (exame médico), os sujeitos foram avaliados fisicamente (medidas de composição corporal e antropométricas) por intermédio de coleta de dobras cutâneas (escapular, abdômen, supra-ilíaca, tricipital, bicipital, peitoral, médio-axilar, coxa e perna), sendo utilizado adipômetro científico (Cescorf Ltda, Brasil), circunferências corporais (braço, antebraço, coxa, perna, todos direito e esquerdo, abdome e tórax) utilizando trena antropométrica (Sanny, Brasil) com três aferições para cada uma das medidas ${ }^{21}$ e também peso e estatura através de balança mecânica antropométrica (Welmy Ltda, Brasil), respectivamente. O cálculo da composição corporal foi realizado através do protocolo de Jackson \& Pollock de três dobras ${ }^{22}$.

Os sujeitos foram avaliados para determinação do consumo máximo de oxigênio $\left(\mathrm{VO}_{2 \text { máx }}\right.$ e limiares anaeróbios (LAns) visando a mensuração da potência aeróbia, em período vespertino, através de esteira ergométrica INBRASPORT (Modelo ATL, Brasil), analisador de gases VO2000 (INBRASPORT Ltda, Brasil) bem como monitor cardíaco (POLAR, modelo S610i, Proximus Ltda; Finlândia) para registros da frequência cardíaca ao longo dos experimentos. A velocidade de corrida inicial foi de $8 \mathrm{~km} / \mathrm{h}$ com incrementos de $1 \mathrm{~km} / \mathrm{h}$ na intensidade da corrida à cada minuto até a exaustão voluntária dos indivíduos. Durante o teste os sujeitos foram verbalmente incentivados com o objetivo de manter um alto nível de estimulação dos mesmos.

Os limiares anaeróbios (LAn1 e LAn2) foram determinados a partir dos valores médios (correspondentes aos 20 seg finais de cada período de esforço) de equivalentes ventilatórios de $\mathrm{O}_{2}\left(\mathrm{VE} / \mathrm{NO}_{2}\right)$ e $\mathrm{CO}_{2}$ (VE/ $\left.\mathrm{VCO}_{2}\right)$, frações expiradas finais de $\mathrm{O}_{2}(\mathrm{FEO} 2)$ e $\mathrm{CO}_{2}\left(\mathrm{FECO}_{2}\right)$ e quociente respiratório (QR), e expressos em função do $\mathrm{VO}_{2}$ (em $\left.\mathrm{ml} / \mathrm{kg} / \mathrm{min}\right)$. O LAn1 correspondeu ao menor valor de $\mathrm{VENO} \mathrm{N}_{2}$ antes de seu aumento continuado, associado ao início do aumento abrupto e continuado do QR. Já o LAn2 foi encontrado no ponto em que os aumentos de VENO $\mathrm{NE}_{2}, \mathrm{VCO}_{2}$ e $\mathrm{PETO}_{2}$ coincidiram com a queda de $\mathrm{PETCO}_{2}{ }^{23}$. Durante a realização das sessões experimentais, a temperatura e umidade relativa do laboratório foram mantidas ao redor de $22^{\circ} \mathrm{C}$ e $45-60 \%$, respectivamente.

Para determinação da concentração de lactato sanguíneo [SLa], foram coletados dos sujeitos, logo após a chegada dos mesmos ao laboratório e também 5 min após a realização do teste de esforço máximo, 25 $\mu \mathrm{l}$ (microlitros) de sangue, por punção da polpa digital do dedo indicador direito. Para tanto, a coleta foi realizada utilizando-se lactímetro portátil (modelo Accutrend Lactate, Roche Diagnostics GmbH; Alemanha), lancetas Ultra-Fine II e tiras de reação da marca Boehringer Mannheim²4.

A economia de corrida é definida como a relação entre o consumo de oxigênio e a velocidade de corrida a intensidades submáximas, com isto torna-se possível caracterizar as exigências aeróbias da corrida. Ela também indica o perfil aeróbio de um atleta fundamentalmente quando este é relativo ao consumo máximo de oxigênio ${ }^{25}$. No presente estudo foi adotada a velocidade de $14 \mathrm{Km} / \mathrm{h}$, sendo este um valor comum à todos os sujeitos submetidos ao teste.

\section{Questionário WURSS - 21}

O Wisconsin Upper Respiratory Symptom Survey (WURSS-21) é composto por 21 questões do tipo "survey" e tem como objetivo coletar informações relacionadas à saúde, que são negativamente afetadas por resfriados comuns e inclui um item de severidade global, 10 itens baseados em sintomas, nove itens funcionais relacionados à qualidade de vida e um item de modificação geral. Todos os itens são baseados em uma escala "tipo-Likert" de severidade, de 0 a $7^{26}$.

\section{Tratamento Estatístico}

Os resultados apresentados constam como médiatdesvio padrão para variáveis antropométricas (Peso, Estatura, Porcentagem de Gordura Corporal) e variáveis dependentes: Consumo Máximo de Oxigênio (VO2máx), Limiares Anaeróbios (Lans 1 e 2), Concentração de Lactato Sanguíneo [Sla], Economia de Corrida (E.C.14Km/h) e WURSS-21. Para tanto, foi aplicado teste t para variáveis independentes, seguida de teste Shapiro Wilk para normalização dos dados obtidos. O nível de significância adotado foi de $a<0,05$. O tratamento estatístico foi realizado através do software Statistica for Windows (versão 5.0; Statsoft, Inc., Estados Unidos).

\section{RESULTADOS}

Os dados antropométricos deste ensaio clínico constam abaixo e estão apresentados como média e desvio padrão, a saber: Foram avaliados 12 sujeitos, dentre eles seis com dermopigmentação, idade (anos), peso (Kg), estatura (cm) e gordura corporal (\%) de 16,5 $\pm 1,16$; $68,10 \pm 6,83 ; 176,29 \pm 7,71 ; 11,49 \pm 0,58$ respectivamente

A seguir são apresentadas diferenças significativas entre os grupos em relação a [SLa] (concentração de lactato sanguíneo) pré e pós-teste, bem como, tempo de teste (Tabela 1).

Em relação às demais variáveis dependentes, não foram encontradas diferenças estatisticamente significativas (Tabela 2).

Tabela 1. Valores expressos como média \pm desvio padrão, referentes à [SLa] Pré, Pós ( $\mathrm{mmol} / \mathrm{L}$ ) e Tempo de Teste (min).

\begin{tabular}{|c|c|c|c|}
\hline Indivíduos $(\mathrm{n}=12)$ & $\begin{array}{l}\text { [SLa] Pré } \\
\text { (mmol/L) }\end{array}$ & $\begin{array}{l}\text { [SLa] Pós } \\
\text { (mmol/L) }\end{array}$ & $\begin{array}{c}\text { Tempo de Teste } \\
\text { (min) }\end{array}$ \\
\hline Dermopigmentados & $4,60 \pm 0,11^{*}$ & $5,65 \pm 0,49$ & $7 \mathrm{~min} 39 \mathrm{seq} \pm 0,03$ \\
\hline Não dermopigmentados & $3,23 \pm 0,88$ & $13,13 \pm 2,20^{*}$ & $8 \min 47 \mathrm{seg} \pm 0,08^{*}$ \\
\hline
\end{tabular}

Tabela 2. Valores médios \pm desvios padrões relativos, referentes ao consumo máximo de oxigênio $\left(\mathrm{VO}_{2 \text { máx }}\right)$, Limiares Anaeróbios (LAns 1 e $2 \%$ Máx) e Economia de Corrida (E.C.14Km/h).

\begin{tabular}{c|c|c|c|c}
\hline Indivíduos ( $\mathbf{n = 1 2 )}$ & Vo $_{\text {2máx }}$ & LAn1 (\% máx & LAn2 (\% máx $)$ & $\begin{array}{c}\text { E.C.14Km/h } \\
\text { (\%) }\end{array}$ \\
\hline Dermopigmentados & $55,0 \pm 2,20$ & $63,4 \pm 5,25$ & $88,1 \pm 3,59$ & $97,6 \pm 2,6$ \\
\hline Não dermopigmentados & $56,5 \pm 2,19$ & $55,4 \pm 2,66$ & $90,9 \pm 3,29$ & $89,6 \pm 6,0$ \\
\hline
\end{tabular}

Quanto aos resultados da análise do questionário WURSS-21 (Figura 2), estes também apontaram diferença estatisticamente significativa entre os grupos $(p<0,05)$, relativa ao número de ocorrências referentes à infecção do trato respiratório superior (ITRS) com 71\% dos casos (10 ocorrências) para o grupo não dermopigmentado contra 29\% (4 ocorrências) do outro grupo.

Tais resultados apresentam como média \pm desvio padrão, os valores

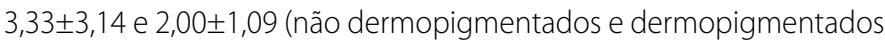
respectivamente).

\section{DISCUSSÃO}

Os resultados do presente estudo demonstraram haver relação entre altas intensidades de esforço e diminuição do desempenho físico, proporcionado pela queda na imunidade. Outros estudos já haviam demonstrado 


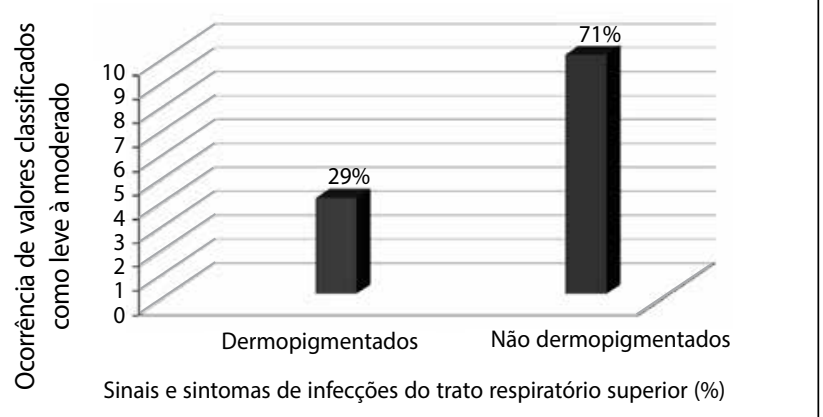

Figura 2. Número de ocorrências de sinais e sintomas referentes a infecções do trato respiratório superior.

que o sistema imune relacionado ao exercício físico como modelo mensurável de indução ao estresse sofre alterações funcionais consideráveis ${ }^{6810,11}$.

Como já visto, o exercício físico de baixa intensidade $\left(<40 \%\right.$ do $\left.\mathrm{VO}_{2 \text { máx }}\right)$ à moderada (entre 40 à $75 \%$ do $\mathrm{VO}_{2 \text { máx }}$ ) proporciona aumento da resposta imune dos mecanismos de defesa do organismo pois eleva as concentrações de glutamina plasmática, sendo este aminoácido produzido pelo metabolismo oxidativo durante atividades com esta intensidade ${ }^{13-17}$.

Já em relação aos exercícios de alta intensidade, alvo deste estudo ( $>65 \%$ do $\mathrm{VO}_{2 \text { máx }}$ ), estes parecem diminuir as respostas imunes devido a glutamina ser convertida em glicose por intermédio do fígado através da neoglicogênese, contribuindo com o metabolismo glicolítico para a manutenção da alta intensidade do esforço, deixando então de atuar junto ao sistema imunológico e por consequência disto, dá-se início ao processo de imunossupressão transitória ${ }^{13,18,19,20}$

Embora exista carência na literatura para explicar tais achados referentes à dermopigmentação, nossos resultados sugerem que o grupo não dermopigmentado obteve [Sla] pré-teste mais baixa, postergando assim o início da fadiga com consequente melhoria na resistência ao teste de esforço máximo. Isto justifica também, uma maior [Sla] no pós-teste deste mesmo grupo devido ao maior tempo do exercício, exigindo dos mesmos, maior esforço.

Os resultados nos levam a interpretar queda na imunidade e consequente menor rendimento em indivíduos com dermopigmentação, não só pelas características do exercício (agudo, de alta intensidade), mas também pelo déficit imunológico possivelmente causado pela ação da dermopigmentação.
Entretanto, em relação as demais variáveis dependentes $\mathrm{VO}_{2 \text { máx }}$ limiares anaeróbios e economia de corrida (Tabela 2), não foram encontradas diferenças estatisticamente significativas. O fato pode ser justificado pelo número reduzido de indivíduos participantes, bem como, escores altos e muito dispersos referentes ao desvio padrão, ocasionando baixo poder ao estudo ${ }^{27}$.

Quanto ao questionário WURSS-21, os resultados indicaram interferência negativa dos sinais e sintomas de ITRS em indivíduos não dermopigmentados. Esta interferência negativa mesmo sendo superior ao grupo dermopigmentado, não afetou o melhor desempenho destes indivíduos durante o teste.

Por outro lado, estes dados sugerem que os indivíduos dermopigmentados, mesmo apresentando sinais e sintomas de infecções do trato respiratório superior (ITRS) inferiores, apresentaram pior desempenho no teste de esforço máximo. Assim, podemos sugerir que, talvez, estes sujeitos já iniciem tais esforços com um déficit imunológico relevante, devido a ação da dermopigmentação.

Vale salientar que a literatura apresenta dados referentes ao WURSS-21 em estudos longitudinais e com ocorrências de sinais e sintomas de ITRS com valores de análise em escala moderada à severa ${ }^{25,26}$, ou seja, propostas diferentes do presente estudo, dificultando demais comparações,

Como limitação deste estudo pode-se citar a ausência de verificação das concentrações de imunoglobulina A (lgA). Notadamente, um importante indicador no diagnóstico da ITRS através da análise salivar dos indivíduos. Com este marcador, poderíamos confirmar com maior precisão a imunossupressão transitória ocorrida em esforços de alta intensidade durante exercício físico agudo 6 . Contudo, o uso do WURSS-21 como ferramenta para monitoramento da ITRS, pôde neste caso, de forma prática e não invasiva, contribuir com tais resultados.

Indubitavelmente, são necessários outros estudos para verificar a contento, a ação da dermopigmentação no exercício intenso.

\section{CONCLUSÃO}

A dermopigmentação produziu rendimento inferior no teste de esforço máximo, influenciando o desempenho no exercício físico.

Todos os autores declararam não haver qualquer potencial conflito de interesses referente a este artigo.

CONTRIBUIÇÕES DOS AUTORES: Cada autor contribuiu individual e significantemente para o desenvolvimento deste artigo. MLM (0000-0002-6593-941x)* e MAU $(0000-0003-4706-774 x)^{*}$ realizaram a redação do manuscrito. Os pesquisadores BLU (0000-0003-4797-3232)* e FBT (0000-0001-7233-5430)* coletaram dados referentes à antropometria dos avaliados. Lucas Duarte Tavares (0000-0001-6032-5251)* e TSCP (0000-0002-9257-2385)* realizaram a coleta de lactato sanguíneo dos indivíduos. MEVP (0000-0003-3450-6088)*e ATB (0000-0003-1994-7619)* realizaram o tratamento estatístico do documento. Todos foram responsáveis pela pesquisa bibliográfica, discussão e revisão do manuscrito, com expressiva contribuição intelectual ao estudo. *Número ORCID (Open Researcher and Contributor ID).

\section{REFERÊNCIAS}

1. Moura Júnior SC, Cortês-Salvio V, Vargas MLC, Leme MP, Rodrigues GP, Babinski MA. Principais efeitos fisiológicos imediatos a aplicação de tatuagens sobre os meridianos de acupuntura. Acta Sci Med. 2009;2(1):29-38.

2. Sacks T, Barcaui CB. Laser e luz pulsada de alta energia- induçaõ e tratamento de reações alérgicas relacionadas a tatuagens. An Bras Dermatol. 2004;79(6):709-14.

3. Sampaio AS, Rivitti EA. Dermatologia. 3a. ed. São Paulo: Artes Medicas; 2008

4. Guyton AC. Fisiologia Humana. 6a. ed, Rio de Janeiro: Guanabara; 1988

5. Guyton AC, Hall JE. Tratado de Fisiologia Médica. 10a. ed. Rio de Janeiro: Guanabara; 2002.

6. Leandro CG, Nascimento E, Manhães-de-Castro R, Duarte JA, de-Castro CM. Exercício físico e sistema imunológico: mecanismos e integrações. Rev Port Cien Desp. 2002;2(5):80-90.

7. Mcardle W, Katch F, Katch V. Fisiologia do exercício: energia, nutrição e desempenho humano. 6a. Ed. Rio de Janeiro: Guanabara; 2008.

8. Hoffman-Goetz L, Pedersen BK. Exercise and the immune system: a model of the stress response? Immunol Today. 1994;15(8):382-7.

9. Keast D, Cameron K, Morton AR. Exercise and the immune response. Sports Med. 1988:5(4):248-67.

10. Pedersen BK, Kappel M, Klokker M, Nielsen HB, Secher NH. The immune system during exposure to extreme physiologic conditions. Int J Sports Med. 1994;15(Suppl 3):S116-21.

11. Brenner I, Shek PN, Zamecnik J, Shephard RJ. Stress hormones and the immunological responses to heat and exercise. Int J Sports Med. 1998;19(2):130-43.

12. Cannon JG. Exercise and resistance to infection. J Appl Physiol (1985). 1993;74(3):973-81.

13. Nieman DC, Henson DA, Sampson CS, Herring JL, Suttles J, Conley M, et al. The acute immune response to exhaustive resistance exercise. Int J Sports Med. 1995;16(5):322-8.

14. Brines $R$, Hoffman-Goetz $L$, Pedersen BK. Can you exercise to make your immune system fitter? Immunol Today. 1996;17(6):252-4.
15. Smith JA, Gray AB, Pyne DB, Baker MS, Telford RD, Weidemann MJ. Moderate exercise triggers both priming and activation of neutrophil subpopulations. Am J Physiol. 1996;270(4 Pt 2):R838-45.

16. Nieman DC. Is infection risk linked to exercise workload? Med Sci Sports Exerc. 2000;32(7 Suppl):S406-11.

17. Fitzgerald L. Overtraining increases the susceptibility to infection. Int J Sports Med. 1991;12(Suppl 1):S5-8.

18. Mars M, Govender S, Weston A, Naicker V, Chuturgoon A. High intensity exercise: a cause of lymphocyte apoptosis? Biochem Biophys Res Commun. 1998;249(2):366-70.

19. Nieman DC, Johanssen LM, Lee JW, Arabatzis K. Infectious episodes in runners before and after the Los Angeles Marathon. J Sports Med Phys Fitness. 1990;30(3):316-28.

20. Petroski EL. Antropometria: técnicas e padronizações. Porto Alegre: Editora Pallotti;1999.

21. Pollock M, Wilmore J. Exercício na saúde e na doença: avaliação e prescrição para prevenção e reabilitação. 2a. ed. São Paulo: Medsi; 1993.

22. Marquezi ML. Bases metabólicas do conceito limiar anaeróbio. Rev Mackenz Educ Físe Esporte 2006;5(2):53-64.

23. Fell JW, Rayfield JM, Gulbin JP, Gaffney PT. Evaluation of the Accusport Lactate Analyser. Int J Sports Med. 1998;19(3):199-204.

24. Santos JAR. Estudo comparativo, fisiológico, antropométrico e motor entre futebolistas de diferentes níveis competitivos. Rev Paul Educ Fís. 1999;13(2):146-59.

25. Moreira A, Borges To, Koslowski Aa, Simões Ac, Barbanti Vj. Esforço percebido, estresse e inflamação do trato respiratório superior em atletas de elite de canoagem. Rev Bras Educ Fís Esporte. 2009;23(4):355-63.

26. Moreira A, Cavazzoni PB. Monitorando o treinamento através do Wisconsin Upper Respiratory Symptom Survey - 21 e Daily Analysis of Life Demands in Athletes nas versões em língua portuguesa. Rev Educ Fis. 2009;20(1):109-19.

27. Thomas JR, Nelson JK, Silverman SJ. Métodos de pesquisa em atividade física. 5a. ed. Porto Alegre: Artmed; 2007 